\title{
Ground Vehicle Guidance along Collision-Free Trajectories using Elastic Bands
}

\author{
Thomas Sattel \\ Heinz Nixdorf Institute \\ Mechatronics and Dynamics \\ University of Paderborn \\ 33102 Paderborn, Germany \\ sattelahni.upb.de
}

\author{
Thorsten Brandt* \\ L-LAB: Public Private Partnership of \\ University of Paderborn and \\ Hella KGaA Hueck \& Co. \\ 33106 Paderborn, Germany \\ thorsten.brandtel-lab.de
}

\begin{abstract}
Latest developments in automotive sensor technology promise an unbroken innovation thrust for driver assistance systems. Accordingly, future vehicles might be able to navigate autonomously. For autonomous vehicles collision avoidance systems (CAS) are essential. Key elements of CAS are automatic path planning, path following and model based estimation of the driving conditions of a CAS-equipped car. For this purpose, a path planning technique using modified elastic bands is presented. Furthermore, a possible overall CAS-structure including path following control and driving condition estimation is discussed. Finally, simulation results for an evasion maneuver are given.
\end{abstract}

\section{INTRODUCTION}

The continuous development of automotive sensor and actuator technology promotes the improvement of existing driver assistance systems and allows the exploration of new applications. Provided that sensor fusion of systems such as radar, lidar, laser scanners and video systems further increases the quality of environmental data in the coming years [1], future vehicles might be able to navigate autonomously. In these autonomous vehicles collision avoidance becomes an essential feature. However, as an intermediate step, collision avoidance systems (CAS) can also be considered for emergency situations that human drivers cannot handle.

A key feature of CAS will be the generation of emergency trajectories. In this paper, a promising path-planning technique is presented, based on elastic bands. This method was introduced by Quinlan and Khatib [2] for robotic path planning. Recently, Hilgert et al. [3] and Gehring and Stein [4] applied the method to automotive vehicle following and lane change maneuver problems, respectively. However, for collision avoidance maneuvers essential modifications are required, as for example in case of sudden environmental changes. These modifications include the influence of the borders of the road and the motions of the obstacles on the deformation of the elastic band. Since a single elastic band can fail to find an existing solution several elastic bands are introduced. Afterwards a criterion is mentioned to choose the final elastic band as emergency trajectory.

As a first approach, path following is accomplished by use of a control system composed of a bicycle model, a

\footnotetext{
*Also with Mechatronics and Dynamics, Heinz Nixdorf Institute
}

linear lateral guidance model and a PID-controller.

Although emergency trajectories can be generated, it cannot generally be guaranteed that a vehicle moves stably on a planned trajectory. Thus, a model based prediction of the driving conditions along the emergency path is carried out. An applicable evaluation method for the stability of the lateral motion of the vehicle based on the Characteristic Velocity Stability Indicator (CVSI), proposed in [5], is mentioned.

The overall structure of a CAS is presented first. Subsequently, path planning and path following are introduced. Finally, sample simulation results for an emergency maneuver are given.

\section{CAS: OVERALL SYSTEM SETUP}

A possible overall setup of a CAS is illustrated in Fig. 1. As soon as the estimated collision risk exceeds a particular threshold value, the planning of an emergency path starts; this point in time is denoted $t_{0}$. The path planning algorithm

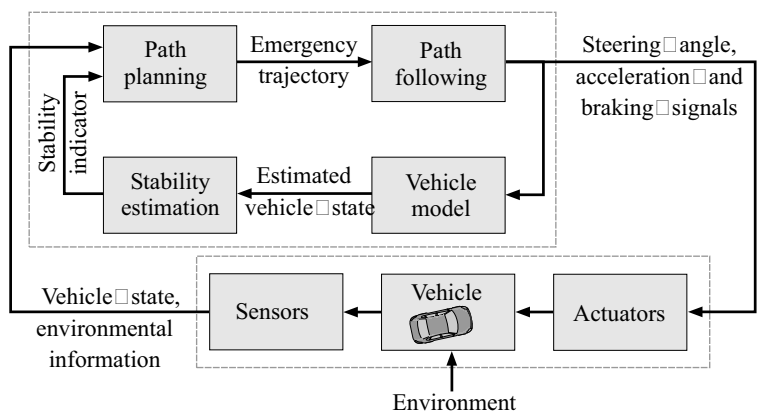

Fig. 1. Possible overall CAS setup

provides possible emergency trajectories based on the available sensor data. In general, the path following controller computes corresponding steering angle, acceleration and braking signals, which allow the vehicle to follow the proposed trajectories. Before these signals are sent to the actuators of the vehicle, the stability of the vehicle for the proposed signals is estimated based on a model, employing the CVSI as shown in Fig. 1. If the CVSI indicates that the vehicle would brake away on the proposed trajectory, an alternative needs to be planned. When a time limit $T_{\max }$ is 
exceeded during this iterative process, emergency braking without steering should be initiated. For drivable emergency trajectories, the corresponding control signals are sent to the actuators. Possible disturbancies in the states of the vehicle or in the environment are detected by sensors and can therefore be incorporated.

\section{PATH PLANNING}

The basic configuration of the path planning approach is illustrated in Fig. 2. Starting from an initially planned path and a corresponding virtual elastic band, emergency trajectories are generated with respect to a vehicle fixed reference frame $\left(\mathbf{a}_{x}, \mathbf{a}_{y}\right)$. The elastic band in Fig. 2, attached to the vehicle, is composed of nodes $P_{i}$ with $i=0, \ldots, N$ connected by linear elastic springs. The node $P_{0}$ is fixed to the CAS-equipped vehicle and the node $P_{N}$ can arbitrarily be placed on the road. The obstacles $O_{j}$ with $j=1, \ldots, M$

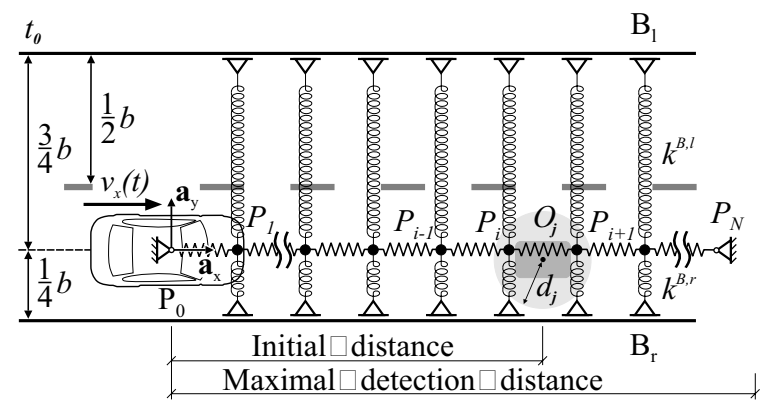

Fig. 2. Initial equilibrium configuration of the elastic band and the virtual potentials of the borders of the road at time $t_{0}$

are modeled by safety circles having diameters $d_{j}$, which should not be penetrated by the path of the center of gravity of the CAS-equipped vehicle. The obstacles $O_{j}$ as well as the borders of the road, $B_{q}$ with $q \in\{l, r\}$, for the left and right border are modeled by repulsive potential fields. The springs between the borders of the road and the nodes $P_{i}$ of the elastic band symbolize the influence of the borders of the road on the elastic band. The initial equilibrium configuration of the elastic band is perturbed by the forces due to the virtual potential fields of the obstacles.

\section{A. Single Elastic Band and External Forces}

Elastic band: The nodes of the elastic band are described in the vehicle fixed reference frame $A$ with unit vectors $\left(\mathbf{a}_{x}, \mathbf{a}_{y}\right)$ as depicted in Fig. 3. Therein, the nodes $P_{i}$ are

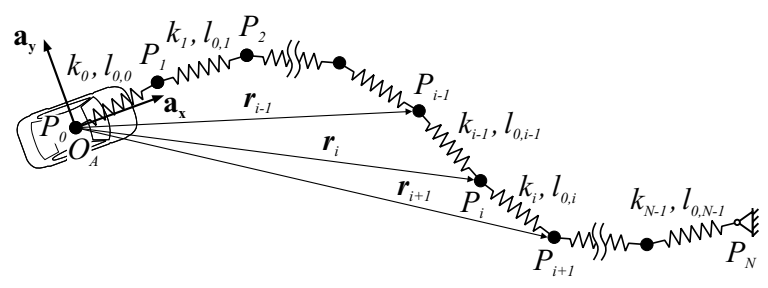

Fig. 3. CAS-equipped vehicle with single elastic band

numbered in temporally ascending order from 0 to $N: t_{i}>$ $t_{i-1}$. The force acting on node $i$ due to a quadratic internal potential $V_{i, i+1}^{\text {int }}$ between the nodes $P_{i}, P_{i+1}$ is given by the directional derivative of this potential

$$
\mathbf{F}_{i, i+1}^{\mathrm{int}}=\frac{\partial V_{i, i+1}^{\mathrm{int}}}{\partial \mathbf{r}_{i}}:=k_{i}\left(\left\|\mathbf{r}_{i+1}-\mathbf{r}_{i}\right\|-l_{0, i}\right) \frac{\mathbf{r}_{i+1}-\mathbf{r}_{i}}{\left\|\mathbf{r}_{i+1}-\mathbf{r}_{i}\right\|},
$$

where $l_{0, i}$ and $k_{i}$ denote the initial spring length and the spring stiffness in interval $i$, respectively. The used Euclidean norm is defined as

$$
\left\|\mathbf{r}_{i+1}-\mathbf{r}_{i}\right\|=\sqrt{\left(r_{i+1, x}-r_{i, x}\right)^{2}+\left(r_{i+1, y}-r_{i, y}\right)^{2}} .
$$

Borders of the road: The virtual potential field of the borders of the road is defined in a way that its absolute value decreases logarithmically towards the middle of the road. The borders are assumed to be continuous and their potentials, $V_{i}^{B_{q}}$ with $q \in\{l, r\}$, are defined separately for the left and the right border. To evaluate the potentials of the left and the right border at the nodes of the elastic band, reference points at the borders having normal vectors pointing toward the corresponding nodes are chosen. The forces acting from the borders on the nodes are calculated by taking the directional derivatives of the logarithmic potential

$$
F_{i}^{B_{q}}=\frac{\partial V_{i}^{B_{q}}}{\partial \mathbf{r}_{i}}:=\frac{k^{B_{q}}}{\left\|\mathbf{r}_{i}-\mathbf{r}_{i}^{B_{q}}\right\|} \cdot \frac{\mathbf{r}_{i}-\mathbf{r}_{i}^{B_{q}}}{\left\|\mathbf{r}_{i}-\mathbf{r}_{i}^{B_{q}}\right\|},
$$

with $k^{B_{q}}$ being the constants of the nonlinear springs as shown in Fig. 2. The vector $\mathbf{r}_{i}^{B_{q}}$ denotes the position vector of the reference point at the border for node $P_{i}$ of the elastic band. In absence of obstacles the equilibrium position of the elastic band can be adjusted at the middle of the right lane. In doing so, force equilibrium $F_{i}^{B_{l}}=F_{i}^{B_{r}}$ yields the relation $\frac{k^{B_{l}}}{k^{B_{r}}}=\frac{0.75 b}{0.25 b}$ between the stiffness constants $k^{B_{l}}$ and $k^{B_{r}}$ for the road geometry given in Fig. 2.

Alternatively, limited forces can be chosen even for small distances to the border

$$
F_{i}^{B_{q}}=k^{B_{q}} \mathrm{e}^{-\left\|\mathbf{r}_{i}-\mathbf{r}_{i}^{B_{q}}\right\|^{2}} \cdot \frac{\mathbf{r}_{i}-\mathbf{r}_{i}^{B_{q}}}{\left\|\mathbf{r}_{i}-\mathbf{r}_{i}^{B_{q}}\right\|}
$$

Obstacles: The diameter $d_{j}$ of the safety circle of obstacle $O_{j}$ consists of the sum of the diameter of the circle that completely covers the obstacle and the width of the CASequipped car. Similar to the borders of the road, the obstacles are modeled by repulsive continuously differentiable potential fields $V^{O_{j}}$. Analogous to the potentials of the borders of the road, potentials of obstacles are modeled as functions that logarithmically decay with the distance to the obstacles. At the beginning of the path planning, $t_{0}$, the velocity of the moving obstacle $O_{j}$ is available based on sensor data. So, the path of the obstacle is estimated for the period of time of the evasion maneuver assuming constant velocity. The path of the obstacle is depicted in Fig. 4. The elastic band is detached from the vehicle and virtually driven through by the vehicle at the planned 


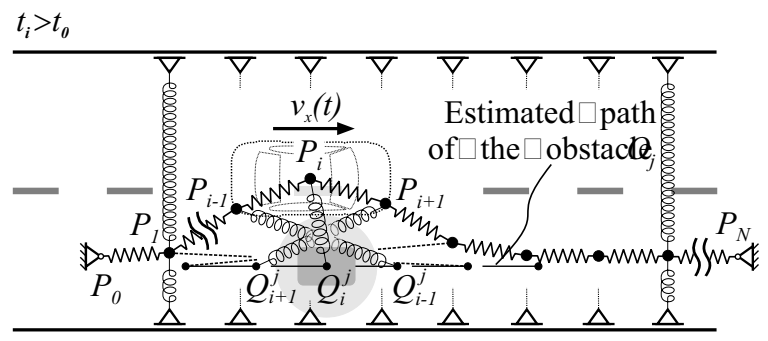

Fig. 4. Incorporation of a moving obstacle

longitudinal velocity $v_{x}(t)$. Thus, each node of the elastic band corresponds to an instant of time $\left(P_{i}, t_{i}\right)$. Points on the estimated path of the moving obstacle $O_{j}$ at the instances of time $t_{i}$ are denoted $Q_{i}^{j}$, yielding $\left(Q_{i}^{j}, t_{i}\right)$. The influence of the moving obstacle on the elastic band is modeled by repulsive forces, acting from $Q_{i}^{j} \rightarrow P_{i}$. For three of these corresponding pairs $\left(Q_{i}^{j}, P_{i}\right)$ this is exemplified by three springs in Fig.4. The forces acting on the nodes of an elastic band are calculated by the directional derivatives of the potentials

$$
F_{i}^{O_{j}}=\frac{\partial V^{O_{j}}\left(\mathbf{r}_{i}\right)}{\partial \mathbf{r}_{i}}=\frac{k^{O_{j}}}{\left\|\mathbf{r}_{i}-\mathbf{r}^{O_{j}}\left(t_{i}\right)\right\|-\frac{d_{j}}{2}} \frac{\mathbf{r}_{i}-\mathbf{r}^{O_{j}}\left(t_{i}\right)}{\left\|\mathbf{r}_{i}-\mathbf{r}^{O_{j}}\left(t_{i}\right)\right\|}
$$

Alternatively, a formulation analogous to (4) for the forces of the obstacles is useful

$$
F_{i}^{O_{j}}=k^{O_{j}} \mathrm{e}^{-\left(\left\|\mathbf{r}_{i}-\mathbf{r}^{O_{j}}\right\|-\frac{d_{j}}{2}\right)^{2}} \cdot \frac{\mathbf{r}_{i}-\mathbf{r}^{O_{j}}}{\left\|\mathbf{r}_{i}-\mathbf{r}^{O_{j}}\right\|} .
$$

\section{B. Equilibrium Solution}

At each node, force equilibrium leads to

$$
\mathbf{F}_{i}^{\mathrm{sum}}=\mathbf{F}_{i, i-1}^{\mathrm{int}}+\mathbf{F}_{i, i+1}^{\mathrm{int}}+\mathbf{F}_{i}^{B_{l}}+\mathbf{F}_{i}^{B_{r}}+\sum_{j=1}^{M} \mathbf{F}_{i}^{O_{j}}=0 .
$$

The forces of the borders of the road and the obstacles are nonlinear. Thus, (7) can only be solved numerically for the position vectors of the nodes of the elastic band. A NewtonRaphson method is employed for this task. In this method the Jacobian of the forces acting on each node

$$
\mathbf{J}=\frac{\partial \mathbf{F}_{i}^{\text {sum }}}{\partial \mathbf{r}_{i}}
$$

is needed. Combining the unknown position vectors of nodes $P_{1}$ to $P_{N-1}$ in one array $\mathbf{q}=\left[\mathbf{r}_{1}, \ldots, \mathbf{r}_{i}, \ldots, \mathbf{r}_{N-1}\right]^{\mathrm{T}}$ and the corresponding forces into one array $\mathbf{F}$, the following system can be set up

$$
\mathbf{J}\left(\mathbf{q}^{\text {old }}\right) \Delta \mathbf{q}=-\mathbf{F}\left(\mathbf{q}^{\text {old }}\right), \quad \mathbf{q}^{\text {new }}=\mathbf{q}^{\text {old }}+\Delta \mathbf{q}
$$

and iteratively solved for $\mathbf{q}$ until all components of $\Delta \mathbf{q}$ are smaller than a value $\varepsilon$. The Newton-Raphson iteration must satisfy two additional constraints. First, it must be enforced that no node leaves the borders of the road or penetrates the safety circle of an obstacle. If a $\Delta \mathbf{q}$ shifts a node into such a non-valid area, the node is still moved along the same displacement vector, but by a smaller distance. Second, it must be guaranteed that the band does not twist. Therefore, if the distance from a node $P_{i+1}$ to node $P_{0}$ is smaller than the distance from node $P_{i}$ to node $P_{0}$ the spring stiffness $k_{i}$ of interval $i$ is increased. Alternatively, the stiffness of all intervals can be increased. It is important to note that after each iteration it has to be determined at which time each node $P_{i}$ is driven through in order to evaluate the positions of the obstacles at the same times, according to the illustration in Fig. 4.

\section{Cubic Spline Interpolation}

The position vectors $\mathbf{r}_{i}$ of nodes $P_{i}$ were already computed by (9). The smooth transition between the nodes of the computed equilibrium configuration of the elastic band is accomplished using cubic splines, similar to [3]. However, the interpolation is modified to account for the proposed collision avoidance approach. According to Fig. 3 the trajectory is described by the position vector $\mathbf{r}(u)$ from $O_{A}$ to a point $P$ on the trajectory, parameterized with the variable $u \in[0,1]$. Thus, for the tag points $\mathbf{r}\left(u_{i}\right)=\mathbf{r}_{i}$ holds, with $0=u_{0}<u_{1}, \ldots,<u_{N}=1$. Between two adjacent nodes $\left(P_{i}, P_{i+1}\right)$ the trajectory is interpolated by

$$
{ }^{i} \mathbf{r}(u)=\mathbf{a}_{i}\left(u-u_{i}\right)^{3}+\mathbf{b}_{i}\left(u-u_{i}\right)^{2}+\mathbf{c}_{i}\left(u-u_{i}\right)+\mathbf{d}_{i}
$$

for the position vector ${ }^{i} \mathbf{r}(u)$ with $u \in\left[u_{i}, u_{i+1}\right]$ in section $i$. The parameters $u_{i}$ are determined according to a centripetal parametrization of the path

$$
u_{i}=\frac{1}{s} \sum_{j=0}^{i-1} \sqrt{\left\|\mathbf{r}_{j+1}-\mathbf{r}_{j}\right\|}, \quad i=1, \ldots, N,
$$

with $s$ being calculated by $s=u_{N}$. The unknown coefficients $\mathbf{a}_{i}, \mathbf{b}_{i}, \mathbf{c}_{i}$ and $\mathbf{d}_{i}$ in (10) are determined from matching conditions and boundary conditions. Between two adjacent nodes the matching conditions are ${ }^{i-1} \mathbf{r}\left(u_{i}\right)={ }^{i} \mathbf{r}\left(u_{i}\right),{ }^{i-1} \mathbf{r}^{\prime}\left(u_{i}\right)=$ ${ }^{i} \mathbf{r}^{\prime}\left(u_{i}\right)$ and ${ }^{i-1} \mathbf{r}^{\prime \prime}\left(u_{i}\right)={ }^{i} \mathbf{r}^{\prime \prime}\left(u_{i}\right)$, where the prime denotes derivation with respect to $u$. For the boundary conditions at $P_{0}$ and $P_{N}$ slopes must be assigned. The composition of the position vectors of all sections yields

$$
\mathbf{r}(u)=\bigcup_{i=0}^{N-1}{ }^{i} \mathbf{r}(u)
$$

For the path following procedure the emergency path description needs to be time-parameterized. For the planned longitudinal velocity $v_{x}=$ constant the time is given by

$$
t(u)=\frac{s(u)}{v_{x}} \quad \text { with } \quad s(u)=\int_{0}^{u}\|\mathbf{r}(\tilde{u})\| \mathrm{d} \tilde{u} .
$$

To achieve the reparametrization $\mathbf{r}(u(t))$, an interpolation is carried out to get the inverse map $u(t)$ from (13).

\section{Several Elastic Bands}

In the previous sections the general procedure of determining collision-free trajectories was developed for one elastic band. Here, this procedure is extended by generating 
several elastic bands. Their equilibrium solutions are determined according to the procedure given in the previous sections. The generation of several bands starts from one initially undisturbed band, as depicted in Fig. 2. All nodes of this initially planned path that lie within the safety circle of an obstacle $O_{j}$ are shifted along the shortest vector into a small neighbourhood outside of the safety circle. However, in general, it cannot be anticipated whether passing an obstacle on the left or on the right is preferable. Therefore, several emergency trajectories, instead of just one, are computed in order to find possible collision-free paths even in complex driving situations. This is illustrated in Fig. 5. To this end, all shifted nodes are reflected across the line pointing from the origin of the vehicle fixed reference frame $O_{A}$ to the center of the corresponding safety circle. For $k$

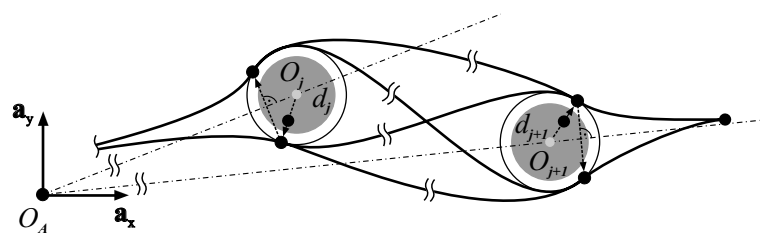

Fig. 5. Several elastic bands avoiding two obstacles $O_{j}$ and $O_{j+1}$ described in a vehicle fixed reference frame $A$

obstacles through which the original trajectory passes, this permutation yields $2^{k}$ elastic bands.

After determining the equilibrium positions and smoothing the $2^{k}$ elastic bands by interpolation with timeparameterized cubic splines, the emergency trajectory with the smallest maximum lateral acceleration is selected. In doing so, the lateral acceleration is estimated by

$$
a_{n}(t)=v_{x}(t)^{2} \kappa(t)
$$

where $\kappa(t)$ denotes the curvature of the emergency trajectory calculated by

$$
\kappa(t)=\frac{\dot{x}_{a} \ddot{y}_{a}-\dot{y}_{a} \ddot{x}_{a}}{\left(\sqrt{\dot{x}_{a}^{2}+\dot{y}_{a}^{2}}\right)^{3}},
$$

using the spline-interpolated, time-parameterized emergency paths $\mathbf{r}(t)=x_{a}(t) \mathbf{a}_{x}+y_{a}(t) \mathbf{a}_{y}$, see (13).

\section{PATH FOLLOWING}

After the final emergency path has been selected, a path following procedure is carried out to determine the steering angle $\delta(t)$ along the emergency path. As a first approach path following is accomplished by use of a control system composed of a bicycle model, a linear lateral guidance model and a PID-controller. The elastic band, virtually lying on the road surface, is now detached from the vehicle model and virtually driven along by the vehicle model at constant longitudinal velocity $v_{x}$.

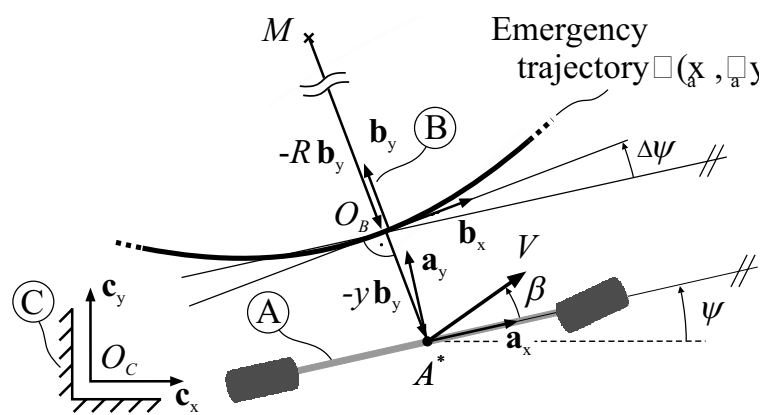

Fig. 6. Bicycle model $A$ with vehicle fixed reference frame $\left(\mathbf{a}_{x}, \mathbf{a}_{y}\right)$ and c.g. $A^{*}$, lateral guidance model $B$ with reference frame $\left(\mathbf{b}_{x}, \mathbf{b}_{y}\right)$ and emergeny trajectory. $\left(\mathbf{c}_{x}, \mathbf{c}_{y}\right)$ represents the earth fixed reference frame

TABLE I

VEHICLE PARAMETERS, [7]

\begin{tabular}{llll}
\hline Symbol & Description & Value & Unit \\
\hline$m$ & Vehicle mass & 1280 & {$[\mathrm{~kg}]$} \\
$J_{z}$ & Moment of inertia about $\mathbf{a}_{z}$-axis & 2500 & {$\left[\mathrm{~kg} \mathrm{~m}^{2}\right]$} \\
$l_{F}$ & Distance of front axle to $A^{*}$ & 1.203 & {$[\mathrm{~m}]$} \\
$l_{R}$ & Distance of rear axle to $A^{*}$ & 1.217 & {$[\mathrm{~m}]$} \\
$c_{\alpha F}$ & Front tire cornering stiffness & 100000 & {$[\mathrm{~N} / \mathrm{rad}]$} \\
$c_{\alpha R}$ & Rear tire cornering stiffness & 100000 & {$[\mathrm{~N} / \mathrm{rad}]$} \\
$i_{s t} l$ & Steering ratio & 20 & {$[-]$}
\end{tabular}

\section{A. Vehicle Model}

A widely used model in lateral vehicle dynamics is the bicycle model $A$ as shown in Fig. 6, see for example [6]. Up to lateral accelerations of $0.4 \mathrm{~g}$, with $g$ being the gravitational constant, linearization of the model about a fixed velocity $V$ provides reliable results. However, for emergency maneuvers it cannot be guaranteed that only low lateral accelerations occur. Therefore, a nonlinear bicycle model is strongly recommended for CAS. For the sake of simplicity the control of the longitudinal motion is avoided by employing a linear bicycle model. The used equations of motion are

$$
\left[\begin{array}{l}
\dot{\beta} \\
\ddot{\psi}
\end{array}\right]=\mathbf{A}\left[\begin{array}{l}
\beta \\
\dot{\psi}
\end{array}\right]+\mathbf{B} \delta
$$

with

$$
\mathbf{A}=\left[\begin{array}{cc}
-\left(\frac{c_{\alpha F}+c_{\alpha R}}{V m}\right) & \left(\frac{-c_{\alpha F} l_{F}+c_{\alpha R} l_{R}}{V^{2} m}-1\right) \\
\left(\frac{-c_{\alpha F} l_{F}+c_{\alpha R} l_{R}}{J_{z}}\right) & -\left(\frac{c_{\alpha F} l_{F}^{2}+c_{\alpha R} l_{R}^{2}}{V J_{z}}\right)
\end{array}\right]
$$

and

$$
\mathbf{B}^{T}=\left[\frac{c_{\alpha F}}{V m}, \frac{c_{\alpha F} l_{F}}{J_{z}}\right] .
$$

The states of the model are the side slip angle $\beta$ and the yaw rate $\dot{\psi}$ as depicted in Fig.,6. The only control input is given by the steering angle $\delta$. The parameters of the simulation model are summarized in Tab.,I.

\section{B. Lateral Guidance Model}

For the vehicle to follow the emergency path automatically, the steering angle along this path has to be deter- 
mined. As a first approach a closed-loop control scheme for lateral guidance is used. Fig. 6 shows the auxiliary reference frame $B$ which follows the emergency trajectory having the $\mathbf{b}_{y}$-axis oriented towards the instantaneous center of rotation $M$ with $R$ being the corresponding radius of curvature. The lateral guidance model is described by the state variables $y$ and $\Delta \psi$, being the lateral distance and the angular deviation between the vehicles longitudinal axis and the tangent on the emergency trajectory, respectively. In the following, the kinematic relations between the lateral guidance state variables $(y, \Delta \psi)$ and the vehicle state variables $(\beta, \dot{\psi})$ are derived. Applying a coordinate transformation between the reference frames $A$ and $B$, the velocity ${ }^{C} \mathbf{v}^{A^{*}}$ of the center of mass $A^{*}$ of the vehicle with respect to the earth fixed reference frame $C$ yields

$$
{ }^{C} \mathbf{v}^{A *}=V \cos (\beta+\Delta \psi) \mathbf{b}_{x}+V \sin (\beta+\Delta \psi) \mathbf{b}_{y} .
$$

The lateral distance speed is given by $\dot{y}={ }^{C} \mathbf{v}^{A *} \cdot \mathbf{b}_{y}$. Assuming small angles results in

$$
\dot{y}=V(\beta+\Delta \psi) .
$$

Next, the kinematic equation for the angular deviation rate is derived. The angular velocity of the lateral guidance model $B$ with respect to the earth fixed reference frame $C$ is given by ${ }^{C} \omega^{B}=(\dot{\psi}+\Delta \dot{\psi}) \mathbf{b}_{z}$. The velocity of the origin $O_{B}$ of the reference frame $B$ with respect to the earth fixed reference frame $C$ is given by

$$
{ }^{C} \mathbf{v}^{O_{B}}={ }^{C} \omega^{B} \times(R+y)\left(-\mathbf{b}_{y}\right)=(\dot{\psi}+\Delta \dot{\psi})(R+y) \mathbf{b}_{x}
$$

and describes the tangential velocity with respect to the emergency path. Setting the $\mathbf{b}_{x}$-component of the velocity $C_{\mathbf{v}} \mathbf{v}^{A *},(19)$, equal to the velocity ${ }^{C} \mathbf{v}_{B}{ }_{B}$ yields

$$
\Delta \dot{\psi}=V \kappa-\dot{\psi},
$$

for $|y| \ll R,|\beta+\Delta \psi| \ll 1$ and the curvature $\kappa=1 / R$, given by (15). The lateral guidance model is given by (20) and (22). Both linear equations can for example be found in [8].

\section{Steering Angle Determination}

The four coupled linear ODEs (16), (20) and (22) are transformed into the frequency domain and rearranged to yield the transfer function structure as illustrated in Fig. 7. The transfer functions $G_{G i}(s)$ with $i \in\{\kappa, \beta, \psi\}$ of the lateral guidance model are derived by inserting (22) into (20). The transfer functions $G_{V i}(s)$ with $i \in\{\beta, \psi\}$ of the vehicle model are obtained by substituting the first equation of (16) into the second equation of (16) and vice versa. The control system output and reference input in Fig. 7, $y_{a}$ and $y_{d}$, denote the actual lateral distance and the desired lateral distance, respectively. In this control structure, the plant transfer function reads $G_{p}=G_{V \beta} G_{G \beta}+G_{V \psi} G_{G \psi}$. As can be readily seen from the transfer function

$$
y_{a}=\frac{G_{c} G_{p}}{1+G_{c} G_{p}} y_{d}+\frac{G_{G \kappa}}{1+G_{c} G_{p}} \kappa
$$

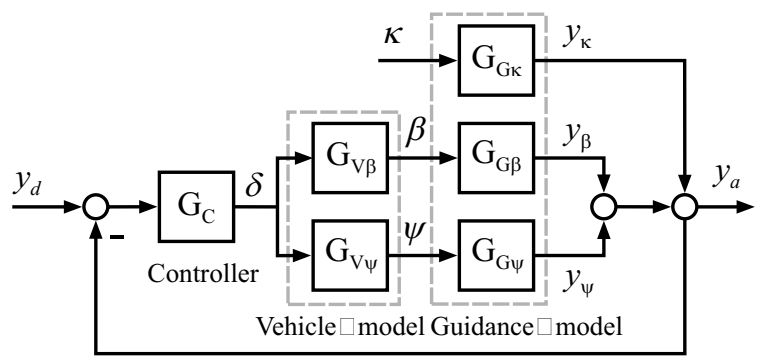

Fig. 7. Control system for lateral guidance vehicle

TABLE II

INTERPRETATION OF CVSI VALUES

\begin{tabular}{ll}
\hline CVSI & Driving condition \\
\hline 1 & Under-steering, stable \\
2 & Neutral-steering, stable \\
3 & Over-steering, stable \\
4 & High-over-steering, indifferent \\
5 & Braking away, unstable
\end{tabular}

of the closed-loop structure, the curvature $\kappa$ of the emergency path acts as a disturbance for the lateral guidance system. Therein, the curvature $\kappa$ is evaluated using (15). For the reference input $y_{d}=0$ is prescribed. A PID-controller is used to follow the planned path, yielding the steering angle. The parameters of the controller are adjusted to reduce oscillating behavior of the disturbance transfer function and thus of the steering angle. More sophisticated path following controllers can be found in [9], [10].

\section{Stability Estimation}

The stability of the lateral motion is estimated by the CVSI, see [5]. The CVSI is determined by comparisons of the longitudinal velocity of the vehicle, $v_{x}(t)$, and its characteristic velocity

$$
v_{c h}^{2}(t):=-\frac{v_{x}^{2}(t)}{1-\frac{\delta(t) v_{x}(t)}{\dot{\psi}(t) i_{s t} l}}
$$

along the emergeny path. The steering angle $\delta(t)$ and the states of the vehicle are previously computed in the path following procedure. The corresponding driving conditions to the CVSI values are given in Tab. II.

\section{SIMULATION RESULTS}

In the simulated scenario a CAS-equipped car drives at a speed of $15 \mathrm{~m} / \mathrm{s}$ on a $7 \mathrm{~m}$ wide road. At time $t_{0}$ a nonmoving obstacle appears $67 \mathrm{~m}$ ahead. In the same lane, an oncoming vehicle travels at $25 \mathrm{~m} / \mathrm{s}$ from an initial distance of about $65 \mathrm{~m}$. The simulation parameters for the path planning are summarized in Tab. III. The forces of the borders of the road and the obstacles were modeled according to (4) and (6), respectively. In Fig. 8 the results for the steering angle, the yaw rate, the lateral acceleration and the CVSI of the CAS-equipped vehicle are graphed. The computed CVSI indicates that the vehicle moves stably along the emergency 
TABLE III

PATH PLANNING PARAMETERS

\begin{tabular}{llll}
\hline Symbol & Description & Value & Unit \\
\hline$d_{1}$ & Safety diameter, oncoming vehicle & 4 & {$[\mathrm{~m}]$} \\
$d_{2}$ & Safety diameter, non-moving obstacle & 3.6 & {$[\mathrm{~m}]$} \\
$k_{1}^{O}$ & Force scaling factor, oncoming vehicle & 8 & {$[-]$} \\
$k_{2}^{O}$ & Force scaling factor, non-moving & 6 & {$[-]$} \\
$\quad$ & obstacle & & \\
$k^{B_{l}}$ & Force scaling factor of left border & 10 & {$[-]$} \\
$k^{B_{r}}$ & Force scaling factor of right border & $10 \cdot \mathrm{e}^{-24.5}$ & {$[-]$} \\
$k_{i}$ & Initial internal spring stiffness for all & 53 & {$[\mathrm{~N} / \mathrm{m}]$} \\
& intervals & & \\
$l_{0, i}$ & Initial length of internal springs & 0.77 & {$[\mathrm{~m}]$} \\
$n$ & Number of nodes & 53 & {$[-]$}
\end{tabular}
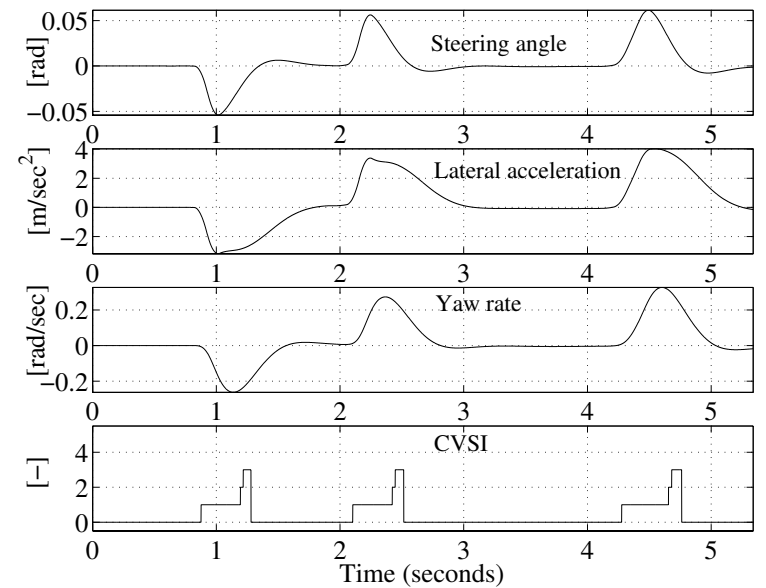

Fig. 8. Steering angle, yaw rate, lateral acceleration and CVSI of the CAS-equipped vehicle following the emergency trajectory based on a linear bicycle model. Initial conditions: $\beta(0)=0, \dot{\psi}(0)=0$

trajectory. The simulated evasion maneuver is illustrated in Fig. 9. The CAS-equipped vehicle and the oncoming vehicle are displayed at corresponding times. Therein, the oncoming vehicle and the non-moving obstacle are represented by their safety circles.

\section{CONCLUSIONS}

A possible overall system setup for a CAS was presented. Several essential modifications of elastic bands were introduced for the path planning in complex driving situations. Accordingly, it was presented how several elastic bands can be generated intermediately and a single solution can be selected afterwards. Also the modeling of the borders of the road and moving obstacles by virtual nonlinear fields was illustrated. Path following was performed using a linear vehicle and lateral guidance model within a feedback control system. The stability of a CAS-equipped vehicle traveling on an emergency trajectory generated by use of elastic bands was estimated with the Characteristic Velocity Stability Indicator (CVSI). Finally, simulation results for a sample emergency maneuver were given.

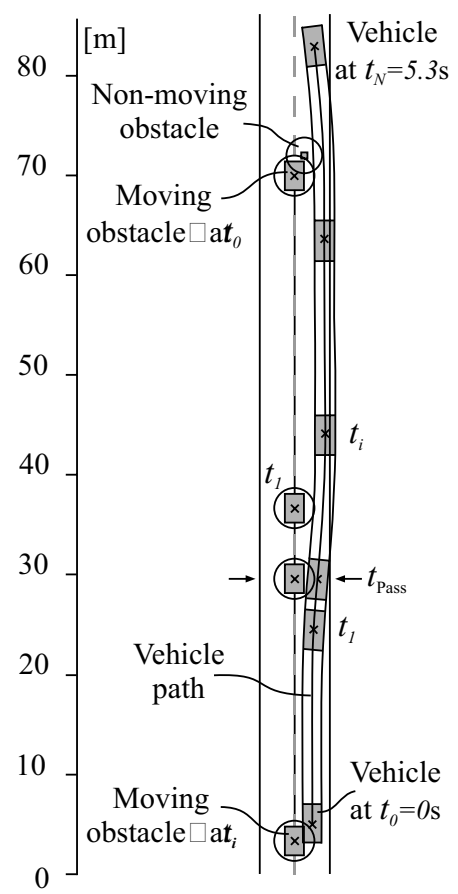

Fig. 9. Simulation results of evasion scenario

\section{ACKNOWLEDGEMENTS}

Many thanks to Hella KGaA Hueck \& Co., the L-LAB and the International Graduate School of Dynamic Intelligent Systems of the University of Paderborn for supporting this work with facilities and a research scholarship.

\section{REFERENCES}

[1] Vukotich, A. and Kirchner, A. (2001). Sensorfusion für Fahrerassistenzsysteme. VDI Berichte 1646, pp. 857, VDI-Verlag, Düsseldorf, Germany.

[2] Quinlan, S. and Khatib, O. (1993). Elastic Bands: Connecting Path Planning and Control. IEEE International Conference on Robotics and Automation, vol. 2, pp. 802-807, Atlanta (GA), USA

[3] Hilgert, J., Hirsch, K., Bertram, T. and Hiller, M. (2003). Emergency Path Planning for Autonomous Vehicles Using Elastic Band Theory. IEEE/ASME International Conference on Advanced Intelligent Mechatronics AIM 2003, July 20-24, Kobe, Japan.

[4] Gehring, S.K. and Stein, F.J. (2001). Elastic Bands to Enhance Vehicle Following. IEEE Intelligent Transportation Systems Conference Proceedings, Oakland (CA), USA

[5] Börner, M., Andréani, L., Albertos, P. and Isermann, R. (2002). Detection of Lateral Vehicle Driving Conditions Based on the Characteristic Velocity. IFAC World Congress 2002, 21.-26. July, Barcelona, Spain.

[6] Isermann, R. (2001). Diagnosis Methods for Electronic Controlled Vehicles. Vehicle Systems Dynamics, Vol. 36, No. 2-3, pp. 77-117

[7] Smith D.E. and Starkey, J.M. (1995). Effects of Model Complexity on the Performance of Automated Vehicle Steering Controllers: Model Development, Validation and Comparison. Vehicle Systems Dynamics, Vol. 24, pp. 163-181

[8] Sönitz, I. (2001). Querregelung eines autonomen Straßenfahrzeugs, VDI Fortschrittsberichte Reihe 8 Nr. 882, VDI-Verlag, Düsseldorf, Germany

[9] Freund, E., Mayr, R. (1997). Nonlinear Path Control in Automated Vehicle Guidance, IEEE Trans. Robot. Autom., Vol.13, No.1, February 1997

[10] Consolini, L., Piazzi, A., Tosques, M. (2001). Motion planning for steering car-like vehicles, Proceedings of the European Control Conference, pp. 1834-1839. 